\title{
Contributions of incidence and case fatality to mortality from bladder cancer in the South Thames Regions
}

\author{
Alison Walker, Ann Petruckevitch, Heather Bourne, Peter Burney
}

\begin{abstract}
Study objective-The aim was to assess the individual contributions of incidence and case fatality to variations in bladder cancer mortality between districts in the South Thames Regions.

Design-The standardised mortality ratios for bladder cancer were calculated for the health districts in the South Thames Regions using data from the Thames Cancer Registry. The results were compared with the standarised registration ratios, used as a measurement of incidence, and survival hazard function, used as a measurement of case fatality. As one determinant of case fatality is the severity at presentation, mortality was also compared with the standardised proportion of cases with advanced disease at diagnosis.
\end{abstract}

Setting-The study took place in the 28 health districts in South East and South West Thames regional health authorities.

Subjects-Subjects were 3271 cases of bladder cancer aged 15-74 years resident in one of the two South Thames regional health authorities at diagnosis and registered between 1982 and 1985 .

Main results-The standardised mortality ratios for the different districts varied from 62 to 139. The standardised registration ratios, the survival hazard functions adjusted for age and stage of disease at diagnosis, and the proportion of cases with severe disease at diagnosis were all independently and significantly related to the standardised mortality ratios in each district.

Conclusions-Mortality from bladder cancer was significantly related to measurements of incidence, case fatality, and severity at presentation. These relations have implications for the health services. The incidence of disease may be modified through preventative measures, the case fatality through improved quality of care, and severity at presentation possibly through prompt management of patients with haematuria. Further studies are needed to investigate why these factors have high values in some districts.

f Epidemiol Community Health 1992; 46: 387-389

Mortality data have already been recognised as a useful source of information for monitoring performance in health services. Rutstein et al used standardised mortality ratios for cause of death considered to be avoidable through timely and appropriate medical intervention to measure quality of care. ${ }^{1}$ Since then standardised mortality ratios have been used to compare the quality of care between different health districts in England and Wales ${ }^{2}$ and between countries in Europe. ${ }^{3}$

The standardised mortality ratio is, however, a summary measure reflecting other variables which affect the mortality rate in a particular area. These include the local incidence of the disease, and the case fatality rate. Futhermore, case fatality is in turn partly determined by the stage of disease at presentation. By measuring these variables some indication may be obtained as to whether they make an important contribution to the ratio.

Information on cancer deaths can be obtained from cancer registry data. The number of registrations at the registry can be used as an estimate of incidence, and survival of registered cases from the date of diagnosis can be used as a measurement of case fatality. The proportion of cases with advanced disease at diagnosis may indicate severity of the disease. Other potentially confounding factors such as age, sex, morphology, and year of diagnosis can also be obtained from the cancer registry. In order to explore the use of these data, details of bladder cancer registrations in South East Thames and South West Thames health regions were chosen for analysis. Bladder cancer was selected since prompt investigation of symptoms, timely surgical management, and close follow up, should substantially reduce mortality. ${ }^{4}$

\section{Methods}

DATA SOURCE

The Thames Cancer Registry provided details of bladder cancer registrations for patients resident at the time of diagnosis in the 28 districts in the South East Thames and South West Thames health regions for the years 1982 to 1985 . Cases used were aged 15 to 74 years at diagnosis. An upper age limit was set, firstly because death certification is less accurate in the elderly, ${ }^{5}$ and secondly because survival is worse in the elderly with a history of bladder cancer than in younger age groups, ${ }^{6}$ and possibly less easily prevented. Details of registrations were provided by health district of residence, and included date of birth, date of last follow up, whether the patient was alive or dead on that date, date of death where appropriate, tumour stage and tumour morphology at diagnosis, sex, and date of diagnosis. Deaths were assigned to the district of residence at diagnosis, not the district of death. Details of the mid-year population in the 28 health districts were obtained from the regional health authorities. 
DATA ANALYSIS

The standardised mortality ratios for bladder cancer cases were calculated from the registry data by comparison of the observed numbers of deaths from all causes among bladder cancer cases in each of the 28 districts with the numbers expected, calculated from the age specific death rates for the total population of the two South Thames regions. A 10 year age stratification was used.

Incidence was measured by calculating the standardised registration ratios for each district. This was carried out in a similar way to the standardised mortality ratios but registrations were substituted for deaths in the calculations.

Cox's proportional hazards model was used to measure survival from diagnosis. ${ }^{7}$ The survival hazard function was then used as a measurement of case fatality. The dates of first diagnosis and of death (if any) were supplied by the registry. Deaths from all causes were used in the analysis. Follow up for patients not known to have died were censored on 31 December 1985 or on reaching the age of 75, whichever was sooner. All deaths were included in the analysis. Survival by district was analysed while controlling for the differences in the distribution of age at diagnosis, sex, year of diagnosis, and stage. Stage was categorised as local, local extension/node involvement, and metastases. The cases with an unclassified stage were excluded from the survival analysis. A backward elimination approach was used to assess the effect that the explanatory variables made on survival, and the likelihood ratio test was used to determine whether they significantly improved the fit of the model.

Severity at presentation was analysed by examnining cases registered with a tumour at an advanced stage at diagnosis-those cases with local extension/node involvement or with metastases. They were standardised (standardised advanced stage ratio) by comparing the observed numbers registered with advanced tumours in each district with the numbers expected. These

Table I Details of cases of bladder cancer in the 28 districts of the South East Thames and South West Thames Regional Health Authorities registered between 1982 and 1985

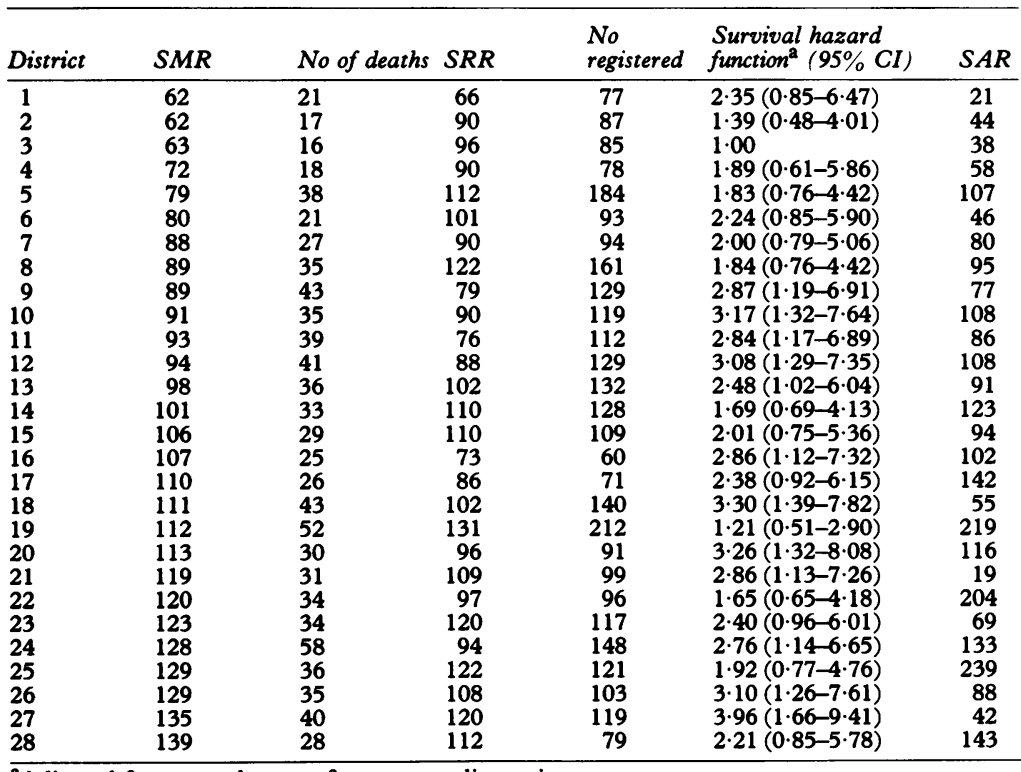

${ }^{2}$ Adjusted for age and stage of tumour at diagnosis

$S M R=$ standardised mortality ratio; $S R R=$ standardised registration ratio; $S A R=$ standardised advanced stage registration ratio (see Methods); $\mathrm{CI}=$ confidence interval were calculated from the age specific registration rates for advanced stage tumours in the two South Thames regions as a whole. Again a 10 year age stratification was used.

\section{Results}

A total of 3385 cases of bladder cancer were registered in residents in the South East Thames and South West Thames health regions between 1982 and 1985, of whom 3271 were aged between 15 and 74 years (inclusive). Most of the cases were elderly, with $58 \%$ of them in the age range of 65 to 74 years. Stage at diagnosis was unevenly distributed between the cases, with $70.1 \%$ having only local disease, $8.6 \%$ having local extension/node involvement, and $3.4 \%$ already having metastases. Those with an unclassified stage accounted for $17.9 \%$. Sex was also unevenly distributed, with $2558(75 \cdot 6 \%)$ men and only $827(24.4 \%)$ women being registered.

Table I shows the variation in standardised mortality ratios among the health districts together with their corresponding values of standardised registration ratios, risk of death, and standardised advanced stage ratio.

The survival hazard function was adjusted for stage and age. Those cases with local extension or node involvement had a risk of death $4.21(95 \%$ confidence interval $3 \cdot 41-5 \cdot 19)$ times greater, and those with metastases $7.91(95 \%$ CI 6.10-10.26) times greater than those with local disease. The risk of death also increased with age at diagnosis by $4 \%$ per year $(95 \%$ CI $3 \%-5 \%)$. Year of diagnosis, morphology, and sex did not affect survival significantly, and were not used to adjust the survival coefficients for the districts.

Table II Association of explanatory variables with the standardised mortality ratio (SMR) as dependent variable in univariate and multiple regression

\begin{tabular}{lcc}
\hline $\begin{array}{l}\text { Independent } \\
\text { variable }\end{array}$ & $\begin{array}{l}\text { Univariate regression } \\
\text { coefficient }(95 \% \text { MItivariate regression } \\
\text { coefficient }(95 \% \text { CI })\end{array}$ \\
\hline SRR & $0.67(0 \cdot 19-1 \cdot 15) \dagger$ & $0.62(0.29-0.94) \ddagger$ \\
Survival hazard & & \\
function & $13.27(1 \cdot 72-24.82) \star$ & $20.29(13.04-27.54) \ddagger$ \\
SAR & $0.19(0.05-0.34) \dagger$ & $0.20(0.10-0.30) \ddagger$ \\
\hline CI = confidence interval; SRR = standardised registration
\end{tabular}
$\mathrm{CI}=$ confidence interval; $\mathrm{SRR}=$ standardised registration ${ }^{\star} \mathrm{p}<0.05 ; \mathrm{tp}<0.01 ; \neq \mathrm{p}<0.001$

Table II shows the univariate and multiple regressions for the relations between the standardised mortality ratio and the standardised registration ratio, the risk of death, and the standardised advanced stage ratio. The standardised mortality ratio was associated with all three variables in both the univariate and multiple regressions.

\section{Discussion}

Cancer registration data may be used to construct several indices which appear to be relevant in considering mortality rates at district level. Although cancer registration data achieve a higher standard of accuracy than routine hospital statistics, the possibility of bias due to incompleteness or inaccuracy needs to be considered.

Variation between districts in the completeness of registration could bias the standardised regi- 
stration ratios. The standardised mortality ratios in contrast are based on death data, which are more likely to be complete. The similarity in the distribution of values of the two ratios, however, suggests that the completeness of registration varies little between districts. Although the registration data include the deaths from bladder cancer they are a relatively small portion of the registrations and are unlikely to account for the association.

Similarly, variations in the acccuracy of recording stage could bias the standardised advanced stage ratios. Failure to record node involvement or metastases would have a large effect because of the small numbers with advanced disease. However, for several other tumour sites, the staging information collected by the registry is related to survival (unpublished data). Further studies to estimate the completeness and accuracy of bladder cancer registration are in progress.

The standardised mortality ratios calculated for each district are a measure of the fatality among bladder cancer cases diagnosed within that district. Although it is possible for patients to have moved between districts, it is extremely unlikely that they would have been resident in one area at the time of diagnosis and have their initial treatment while resident in another.

The standardised mortality ratios calculated for each district are defined differently to those published by the Office of Population Censuses and Surveys (OPCS). The data on which the OPCS mortality statistics are based are categorised by the area of death. The focus of this paper, however, is the explanatory factors for the fatality of cases first diagnosed in a given district. The standardised mortality ratios used were thus categorised by the district of diagnosis. One advantage of this over the conventional ratio is that it is not biased by the distribution of nursing homes and retirement areas, which tend to distort the values for small areas based on place of death.

The standardised mortality ratio for an "avoidable death" such as bladder cancer has already been used as an indicator of possible shortcomings in health care provided in a district. ${ }^{2}$ The ratio will be determined by the age specific incidence and case fatality, which will in turn be partly determined by the severity at diagnosis. This study used cancer registry data to estimate the effects of these three factors. All were found to be significantly and independently related to the ratio. An understanding of why they have high values in some districts may help to direct improvements in local health services and thereby lower mortality.

The variation in incidence between the districts may be affected by local industrial exposure, especially to aniline dyes, ${ }^{13}$ and by local differences in smoking habits. ${ }^{14}$ It may be possible for these to be modified by preventive measures including control of exposure and screening in the workplace and health education. Delay in starting treatment may also contribute to a high mortality. Wallace and Harris showed that pretreatment delay was associated with poor survival for early invasive bladder cancer, the three year survival rate decreasing from $60 \%$ to $25 \%$ when treatment was delayed by more than one month but less than six months. ${ }^{4}$ Delay had little effect on survival for superficial tumours. They recommended that all cases of haematuria, the presenting symptom in $80 \%$ of the cases of bladder cancer, should be investigated promptly. Several studies have attempted to account for the delay in treating bladder cancer. ${ }^{489}$ The longest delays have been attributed to hospitals, and in particular to time spent on waiting lists. Interhospital differences in delay have also been found. ${ }^{9}$

There may be other factors associated with case fatality which are currently hard to quantify. A small study in West Yorkshire carried out to explain the high mortality in Huddersfield (standardised mortality ratio $=244$ ) compared to Bradford $(S M R=80)$ concluded that the tumours in Huddersfield were more aggressive. ${ }^{10}$ It showed that survival of superficial cases of bladder cancer in Huddersfield was worse than in Bradford despite earlier presentation in Huddersfield. The uncertain natural history of bladder tumours has been documented before. ${ }^{11}$ More than $70 \%$ are superficial at presentation and $80 \%$ of these remain confined to the mucosa and submucosa. Others that appear morphologically identical become invasive or metastasise.

Further work is in progress to describe in detail any association between method of management and survival with bladder cancer.

Miss Petruckevitch is supported by the Department of Health. The authors are grateful to Dr W Stewart for discussions during the course of this research, to Professor Jocelyn Chamberlain, Dr Janine Bell, and Dr Martin Gulliford for their comments on a previous draft, and to Professor W W Holland for his support.

1 Rutstein D, Berenberg W, Chalmers T, et al. Measuring the quality of medical care. $N$ Engl f Med 1976; 294: 582-8.

2 Charlton JRH, Hartley RM, Silver R, Holland WW. Geographical variation in mortality from condition amenable to medical intervention in England and Wales. Lancet 1983; i: 691-6.

3 Holland WW (ed). European Community atlas of avoidable mortality. Oxford: Oxford University Press, 1988

4 Wallace DM, Harris DL. Delay in treating bladder tumours. Lancet 1965; ii: 332-4.

5 Alderson $M$. International mortality statistics. London Macmillan, 1981: 14-34.

6 Narayana AS, Loening SA, Slymen DJ, Culp DA. Bladder cancer: factors affecting survival. $\mathcal{f}$ Urol $1983 ; 130: 56-60$ 7 Cox D. Regression models and life tables. $\mathcal{F} R$ Stat Soc $B$ 1972; 74: 187-220.

8 Stower MJ. Delays in diagnosing and treating bladder cancer. $B M \mathcal{F}$ 1988; 296: 1228-9.

9 Macarthur C, Pendleton LL, Smith A. Treatment delay in patients with bladder tumours. I Epidemiol Community Health 1985; 39: 63-6.

10 Cartwright RA, Glashan RW, Gray B. Survival of transitional cell carcinoma cases in two Yorkshire centres. Br f Urol 1980; 52: 497-9.

11 Raghavan D, Shipley WU, Garnick MB, Russell PJ, Richie $\mathrm{JP}$. Biology and management of bladder cancer. $N$ Engl $\mathcal{F}$ Med 1990; 322: 1129-38.

12 Wallace DMA. Occupational urothelial cancer. Br $f$ Urol 1988; 61: 175-81.

13 Cole P, Monson RR, Haning H, Friedell GH. Smoking and cancer of the lower urinary tract. $N$ Engl $\mathcal{Y}$ Med 1971; 284: 129-34. 